\title{
Implementation of a PID Type Sliding-Mode Controller Design Based on Fractional Order Calculus for Industrial Process System
}

\author{
Kagan Koray Ayten*, Ahmet Dumlu \\ Department of Electrical \& Electronics Engineering, Erzurum Technical University, \\ 25700, Erzurum, Turkey \\ kagan.koray@erzurum.edu.tr
}

\begin{abstract}
This paper is devoted to designing a fractional order Proportional Integral Derivative (PID) type sliding mode control method (FO-PIDSMC) for a non-linear liquid level coupled tank process system. By considering the individual advantages of the FO calculus and PID type SMC method, this proposed FO-PIDSMC technique is designed to integrate the FO calculus method with PID type SMC scheme to obtain an accurate and robust liquid level tracking in terms of the predefined reference trajectory. The real-time experimental results of the proposed controller suggest a dramatic improvement over the traditional process system controller methods in both trajectory tracking and required control action.
\end{abstract}

Index Terms-Sliding mode controller method; Fractionalorder calculus; Process system; Liquid level coupled tank system.

\section{INTRODUCTION}

In many industrial areas, the liquid level control between tanks is a crucial application, such as water distribution [1], biochemical [2], power generation, petrochemical processing [3], and so on. In these applications, the liquid is stored in more than one tank connected with a hole. In this type of system, generally, the electrical pumps are used as actuators to motorize valve systems. Besides, the flowmeter and pressure sensor can assist to obtain a liquid level for control applications. This process system has a nonlinear behavior due to the dynamic parameter variations and pump-valve characteristics. The classical industrial process applications, traditional (Proportional Integral Derivative PID) control methods are beneficial controller methods for linear and basic systems. In spite of this, the classical PID type controller methods are incapable to fulfil the demands of controlling high accurate liquid levels during the application due to the nonlinear behavior of the system. For this purpose, many nonlinear control algorithms have been performed to control this type of system. To solve the problems mentioned above and to provide high precision liquid level control, a nonlinear control method should be used.

In the literature, various non-linear control techniques are performed to liquid level process systems to obtain high precise control, such as adaptive based [4] and fractional

Manuscript received 28 May, 2021; accepted 6 November, 2021. order controller [5]-[7], back-stepping controller [8], a model-based back-stepping controller [9].

Additionally, different types of fuzzy-based controller methods have been considered to obtain accurate control over the system nonlinearities, disturbance, and parameter changes [10]-[12]. Although these are crucial benefits, the fuzzy-based controller efficiency can be disrupted in terms of large delay conditions. Because the Fuzzy logic controller uses trial and error for optimization and has several tuning parameters (fuzzification, defuzzification, and inference). Therefore, it takes some time to set parameters in a real-time application [13].

In the literature, the Sliding Mode Control (SMC) methods have gained attraction recently, especially as a process control algorithm [13], [14]. The crucial benefit of the SMC is an effective and robust control process in terms of the unknown parameters and unmodeled dynamics. In addition to this, by controlling a nonlinear dynamic system in terms of the external disturbances of uncertainties, system states have stayed on the sliding surface with the help of the switching function in SMC [15], [16]. In recently, many types of SMC methods are developed for controlling various types of dynamic systems for instance the terminal SMC [17], the second-order SMC [18], high-order SMC techniques (HOSMC) [19], and the integral SMC (ISMC) [20]. In these types of SMC methods, two different control components have consisted. The first component is called the "phase control law" which is obtained by the approximate model of the endowed system. Another component of the SMC is presented as the "switching control law". This switching control law is composed to guarantee the uncertainties of the system. By combining these parts of the SMC, a very efficient, effective, and robust method can be developed for controlling the process system. In literature, an integral sliding mode controller for heat exchanger process water system is designed by Fan and Yang [21]. The results of their proposed method performed well in uncertainties during the simulated examples. In [22], the terminal SMC has been performed to the complex pneumatic and hydraulic process systems in simulation, as well as in real-time application. Their controller provides avoiding uncertainties by following the expected acceleration time sequence accurately.

In addition to the aforementioned control methods, the 
fractional order (FO) control technique is also a very attractive control method in the literature. In terms of increasing the efficiency of the aforementioned controller techniques, in some studies, fractional-order is considered during the process control to increase the system robustness due to an arbitrary order of the ordinary and the integral calculus [23]-[31].

In this work, a mathematical controller design has been developed for considering the benefits of FO calculus and SMC techniques. The designed FO with PID type SMC technique (FO-PIDSMC) has been performed for controlling the proposed liquid level control system. In literature, the PD-SMC and Fractional-SMC have been theoretically executed in [32]. The proposed technique considers the combination of FO calculus with the PID type SMC model for a liquid level process system for the first time in the literature. The reason for performing fractional-order calculus combined with PID type SMC is to provide fast finite-time convergence, accurate liquid level tracking, chattering free control, and avoiding uncertainty during the real-time process motion. Moreover, the nonlinear dynamic equations of the liquid level coupled tank process system have been analytically obtained. To show the effectiveness of FO-PIDSMC, an experimental application is executed by the conventional Feed Forward-PI (FF-PI) controller technique. The real-time execution and outputs prove that the given FO-PIDSMC technique progressively increases the system's controller efficiency compared to the traditional process control techniques.

The structure of the paper can be given as follows. Section II demonstrates the mathematical modeling of the coupled tank process system structure. The FO calculus method is given in Section III. The PID type second-order SMC design is given in Section IV and the experimental outcomes are executed in Section V. Lastly, the conclusions are given in Section VI.

\section{Dynamic Model OF THE COUPLed TANK SySTEM}

The liquid level coupled tank system is illustrated in Fig. 1. An electrical motor actuates the system's pump. As shown in Fig. 1, Tank 1 and Tank 2 are connected together with an orifice. Additionally, the pressure sensor consists each tank for the level measurement.

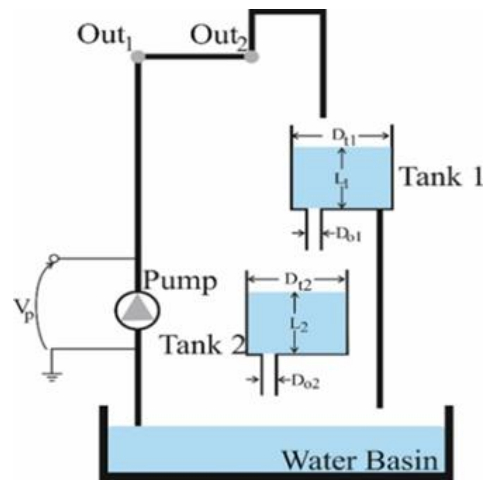

Fig. 1. The liquid level coupled tank system.

So, as to create a mathematical model of the system, firstly, the time derivation of the liquid level can be considered as taken into account the fluid dynamics law as follows

$$
\dot{L}_{i}(t)=\frac{1}{A_{i}}\left(F_{I N_{i}}-F_{O U T_{i}}\right) \quad i=1,2
$$

In (1), $L_{i}(t)$ shows the position of liquid in the tanks $(\mathrm{cm}), \quad F_{I N_{i}}$ and $F_{O U T_{i}}$ are in/out flow water percentages $\left(\mathrm{cm}^{3} / \mathrm{s}\right)$ for the $i^{\text {th }}$ tank. In (1), $\mathrm{A}_{\mathrm{i}}$ shows the cross-sectional area $\left(\mathrm{cm}^{2}\right)$. In fluid dynamics law, $F_{I N_{1}}$ is related to the voltage applied to the actuator of the liquid level system's pump, as shown in (2)

$$
F_{I N_{1}}=K_{p} V_{p}(t)
$$

The pump's constant $\left(\mathrm{cm}^{3} / V s\right)$ is given by $K_{p}$ and the control input (voltage pump $u(t)$ (Volt)) is named by $V_{p}(t)$. Additionally, considering Bernoulli's law, the outflow velocity from the orifice of the individual tanks $v_{\text {OUT }}(t)$ is presented by (3)

$$
v_{O U T_{i}}(t)=\sqrt{2 g L_{i}(t)} .
$$

In this situation, the ach liquid tank's outflow water percentage $\left(F_{O U T_{i}}\right)$ is calculated from (4)

$$
F_{O U T_{i}}(t)=a_{i} \sqrt{2 g L_{i}(t)}
$$

In (4), $g$ shows the gravitational acceleration $\left(981 \mathrm{~cm} / \mathrm{s}^{2}\right)$, and the outflow water orifice's crosssectional area $\left(\mathrm{cm}^{2}\right)$ is presented by $a_{i}$. Due to the system mechanical structure of the liquid system, the inflow water of Tank $2\left(F_{I_{2}}(t)\right)$ should be equal to the outflow of Tank $1\left(F_{\text {OUT }}(t)\right)$ as follows

$$
F_{I N_{2}}(t)=F_{O U T_{1}}(t)
$$

So, in the light of the aforementioned explanation above in (2)-(5), the mathematical background of the fluid dynamics of the liquid level system can be obtained as follows:

$$
\begin{gathered}
\dot{L}_{1}(t)=\frac{K_{p} u(t)-a_{1} \sqrt{2 g L_{1}(t)}}{A_{1}}, \\
\dot{L}_{2}(t)=\frac{a_{1}}{A_{2}} \sqrt{2 g L_{1}(t)}-\frac{a_{2}}{A_{2}} \sqrt{2 g L_{2}(t)} .
\end{gathered}
$$

By taking into account (6) at equilibrium $\left(\dot{L}_{1}(t)=0\right)$, the steady-state pump voltage $\left(u_{1}(t)\right)$ gives $L_{10}$, which describes the steady-state Tank 1's level, and also by considering (7) at equilibrium $\left(\dot{L}_{2}(t)=0\right)$, figures out $L_{10}$ in Tank 1 and this calculation gives $L_{20}$, which describes the requested steady-state constant for Tank 2's level. These 
calculations are given in (8) and (9) as follows:

$$
\begin{gathered}
u_{1}(t)=a_{1} \frac{\sqrt{2 g L_{10}}}{K_{p}}, \\
L_{10}=\left(\frac{a_{2}}{a_{1}}\right)^{2} L_{20} .
\end{gathered}
$$

After all these calculations, the deviation variables can be defined by considering the operating range in terms of the small departure voltages $\left(u_{1}(t)\right)$ and heights $\left(L_{1}(t), L_{2}(t)\right)$ as follows:

$$
\left\{\begin{array}{l}
L_{11}(t)=L_{1}(t)-L_{10}, \\
L_{21}(t)=L_{2}(t)-L_{20}, \\
u_{2}(t)=u(t)-u_{1}(t) .
\end{array}\right.
$$

The second-order SMC can be defined as $u_{2}(t)$. Therefore, (6) and (7) can be converted as follows:

$$
\begin{gathered}
\dot{L}_{1}(t)=-\frac{a_{1}}{A_{1}} \sqrt{2 g\left(L_{10}+L_{11}(t)\right)}+ \\
+\frac{K_{p}}{A_{1}}\left(u_{1}(t)+u_{2}(t)\right), \\
\dot{L}_{2}(t)=-\frac{a_{1}}{A_{2}} \sqrt{2 g\left(L_{11}(t)+L_{10}\right)}- \\
-\frac{a_{2}}{A_{2}} \sqrt{2 g\left(L_{21}(t)+L_{20}\right)} .
\end{gathered}
$$

As it is seen from (11) and (12), the couple tank system has nonlinear dynamic equations due to variations of the system's parameter, valve, and pump characteristics. Hence the proposed SMC to the proposed model performs the linearization of the desired system model based on an operating point $\left(L_{10}, u(t)\right)=\left(L_{10}, u_{1}(t)\right)$. Considering the first-order Taylor's series approximation about $\left(L_{20}, L_{10}\right.$, and $\left.u_{1}(t)\right)$ from (11) and (12), it leads us as follows:

$$
\begin{gathered}
\dot{L}_{11}(t)=\alpha_{1} L_{11}(t)+\beta_{1} u_{2}(t), \\
\dot{L}_{21}(t)=\alpha_{2} L_{21}(t)+\beta_{2} L_{11}(t),
\end{gathered}
$$

where:

$$
\left\{\begin{array}{l}
\alpha_{1} \cong-\frac{a_{1}}{A_{1}} \sqrt{\frac{g}{2 L_{10}}}, \quad \beta_{1} \cong \frac{K_{p}}{A_{1}}, \\
\alpha_{2} \cong-\frac{a_{2}}{A_{2}} \sqrt{\frac{g}{2 L_{20}}}, \beta_{2} \cong \frac{a_{1}}{A_{2}} \sqrt{\frac{g}{2 L_{10}}} .
\end{array}\right.
$$

After all, utilizing (13) and (14), the level control of Tank $2,\left(L_{2}(t)\right)$, is computed. The subsystem out/in flow is given by $L_{21}$ and $L_{11}$, which are used in (14) as an input and output. Additionally, the subsystem dynamics in (13) and (14) are calculated as Laplace transforms of these equations given in (16) and (17):

$$
\begin{aligned}
& G_{1}(s)=\frac{K_{1}}{\tau_{1} s+1}, \\
& G_{2}(s)=\frac{K_{2}}{\tau_{2} s+1},
\end{aligned}
$$

where $K_{1}=\frac{K_{p} \sqrt{2 g L_{10}}}{a_{1} g}, \quad K_{2}=\frac{a_{1} \sqrt{L_{20}}}{a_{2} \sqrt{L_{10}}}, \quad \tau_{1}=\frac{A_{1} \sqrt{2 g L_{10}}}{a_{1} g}$, and $\tau_{2}=\frac{A_{2} \sqrt{2 g L_{20}}}{a_{2} g}$. Equations (16) and (17) can be considered for conventional PI, second-order SMC, or other non-linear control techniques. Table I represents the coupled tank system's parameters.

TABLE I. THE LIQUID LEVEL SYSTEM PARAMETERS.

\begin{tabular}{|c|c|c|}
\hline Description & Symbol & Value\&Units \\
\hline Constant of Pump Flow & $K_{p}$ & $3.3 \mathrm{~cm}^{3} / \mathrm{s} / V$ \\
\hline Gravitational constant & $g$ & $981 \mathrm{~cm} / \mathrm{s}^{2}$ \\
\hline Tank 1\&2 Outlet Areas & $a_{1}=a_{2}$ & $0.1781 \mathrm{~cm}^{2}$ \\
\hline $\begin{array}{c}\text { Tank 1\&2 Inside Cross- } \\
\text { Section Areas }\end{array}$ & $A_{1}=A_{2}$ & $15.5179 \mathrm{~cm}^{2}$ \\
\hline
\end{tabular}

\section{Method OF Fractional ORdER}

In this section, a piece of fundamental information about the FO calculus is given. The Riemann-Liouville definition is the most frequently used in scientific literature. The fractional derivative and integral of $a_{t h}$ order of function $f(t)$ are illustrated by [31]:

$$
\begin{gathered}
\mathcal{D}^{\alpha} f(t)=\frac{d^{\alpha} f(t)}{d t^{\alpha}}=\frac{1}{\Gamma(n-\alpha)}\left(\frac{d^{n}}{d t^{n}}\right) \int_{0}^{t} \frac{f(\tau)}{(t-\tau)^{\alpha+1-n}} d \tau \\
\mathcal{D}^{-\alpha} f(t)=\mathcal{L}^{\alpha} f(t)=\frac{1}{\Gamma(\alpha)} \int_{0}^{t} \frac{f(\tau)}{(t-\tau)^{1-\alpha}} d \tau
\end{gathered}
$$

where $n-1<\alpha<n$ and $n$ show an integer value. $D^{n}$ and $\mathcal{L}^{n}$ demonstrate the fractional derivative and integral part, respectively. Euler's Gamma function, $\Gamma($.$) , is$ presented by

$$
\Gamma(\lambda)=\int_{0}^{\infty} e^{-t} t^{\alpha-1} d t
$$

The $n_{t h}$ order derivative $\left(d^{n} / d t^{n}\right)$ of the fractional derivative operator, $\mathcal{D}^{\alpha} f(t)$, can be turned into as

$$
\frac{d^{n}}{d t^{n}}\left(\mathcal{D}^{\alpha} f(t)\right)=\mathcal{D}^{\alpha}\left(\frac{d^{n} f(t)}{d t^{n}}\right)=\mathcal{D}^{\alpha+n} f(t)
$$

The most important specification of the proposed controller (FO-PIDSMC) is the less sensitivity to variation which occurs to parameters of a liquid level coupled tank system. With the help of FO calculus, more effectiveness and efficiency are provided in the desired controller in terms 
of the arbitrary order of the ordinary derivative and integral computation. The strength of the proposed controller (FOPIDSMC) for the liquid level system is more effective than the FF-PI method in terms of more degrees of freedom (DOF) for setting. The extra 2-DOF in the fractional order controller gives more effectiveness for the setting of the dynamic behavior when compared to the FF-PI technique. So, the FO techniques have a crucial effect on the controller system behavior to decrease the steady-state error and stability.

\section{FO-PID TyPE SECOND-ORDER SLIDING CONTROLLER DESIGN}

In this section, the PID type SMC method is created so as to execute an effective liquid level trajectory tracking in terms of the presence of uncertainties. Figure 2 shows the proposed method block diagram. In this figure, the reference liquid level trajectory is compared with the real-time output of the coupled tank system for computing the error during the execution. In this case, a computed error will be used in a sliding surface function $(S)$, and this function is also used to feed into the FO-PIDSMC.

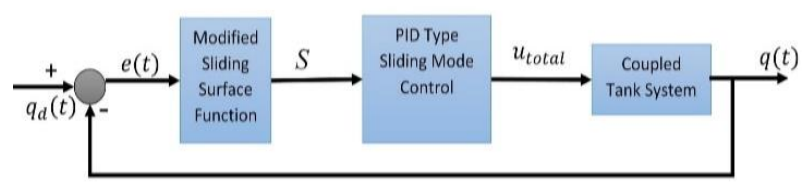

Fig. 2. The proposed FO-PIDSMC block diagram for the proposed system.

In this case, the state variables can be defined as follows:

$$
\begin{aligned}
& \dot{x}_{1}(t)=x_{2}(t)=L_{1}(t), \\
& \dot{x}_{3}(t)=x_{4}(t)=L_{2}(t) .
\end{aligned}
$$

Hence, by taking the inverse Laplace transformation of (16) and (17), the differential equation of the system can be obtained as follows:

$$
\begin{gathered}
x_{1}(t)=-\frac{1}{\tau_{1}} \dot{x}_{1}(t)+\frac{K_{1}}{\tau_{1}} u_{2_{1}}(t), \\
x_{3}(t)=-\frac{1}{\tau_{2}} \dot{x}_{3}(t)+\frac{K_{2}}{\tau_{2}} u_{2_{2}}(t) .
\end{gathered}
$$

The process outputs are given by $x_{2}(t)$ and $x_{4}(t)$, and $u_{2_{1}}(t)$ is equal to the control input of Tank 1 , and $u_{2_{2}}(t)=L_{1}(t)$ are the control inputs of Tank 2. Hence the second-order equation of motion can be obtained in a matrix form as follows

$$
\boldsymbol{x}_{2 x 1}=\boldsymbol{f}(\boldsymbol{x})_{2 x 1}+\boldsymbol{g}(\boldsymbol{x})_{2 x 1} \boldsymbol{u}(\boldsymbol{t})_{2 x 1}+\boldsymbol{\zeta}(\boldsymbol{t}, \boldsymbol{u}(\boldsymbol{t}))_{2 \times 1},
$$

where the bounded lumped uncertainty is given by $\boldsymbol{\zeta}(\boldsymbol{t}, \boldsymbol{u}(\boldsymbol{t}))_{2 \times 1}$. In addition to the aforementioned above, the tracking error $\boldsymbol{e}(\boldsymbol{t})_{2 x 1}$ is given as follows

$$
\boldsymbol{e}(\boldsymbol{t})_{2 \times 1}=\boldsymbol{x}_{d 2 \times 1}-\boldsymbol{x}_{2 \times 1}
$$

The controller is divided into two sub-sections, such as the phase control law and switching feedback law to avoid the limited unknown uncertainty in the system. The designed controller is divided into two sub-sections as the phase control and switching feedback part to meet the limited unknown uncertainty in the system. Equation (28) gives the PID type sliding surface function as follows

$$
\begin{gathered}
\boldsymbol{s}(\boldsymbol{t})_{2 x 1}=\boldsymbol{k}_{p_{2 \times 2}} \boldsymbol{e}(\boldsymbol{t})_{2 \times 1}+\boldsymbol{k}_{i_{2 \times 2}} \int \boldsymbol{e}(\boldsymbol{\tau})_{2 \times 1} d \tau+ \\
+\boldsymbol{k}_{d_{2 \times 2}} \dot{\boldsymbol{e}}(\boldsymbol{t})_{2 \times 1} .
\end{gathered}
$$

In (28), the PID control gain matrix parameters are $\boldsymbol{k}_{p_{2 \times 2}}$, $\boldsymbol{k}_{\boldsymbol{d}_{2 \times 2}}$, and $\boldsymbol{k}_{\boldsymbol{i}_{2 \times 2}}$, respectively. In (28), a FO-PID type sliding surface is created by using an aforementioned FO calculus as follows

$$
\begin{gathered}
\boldsymbol{s}(\boldsymbol{t})_{2 x 1}=\boldsymbol{k}_{p_{2 \times 2}} \boldsymbol{e}(\boldsymbol{t})_{2 x 1}+\boldsymbol{k}_{i_{2 x 2}} \mathcal{D}^{-\alpha}\left\{\boldsymbol{e}(\boldsymbol{t})_{2 x 1}\right\}+ \\
+\boldsymbol{k}_{\boldsymbol{d}_{2 \times 2}} \mathcal{D}^{\alpha}\left\{\boldsymbol{e}(\boldsymbol{t})_{2 x 1}\right\} .
\end{gathered}
$$

To use the error function in the proposed control technique, (30) can be taken when the derivative of (29) is taken twice as follows

$$
\begin{aligned}
\boldsymbol{s}(\boldsymbol{t})_{2 x 1}= & \boldsymbol{k}_{\boldsymbol{p}_{2 \times 2}} \boldsymbol{e}(\boldsymbol{t})_{2 x 1}+\boldsymbol{k}_{i_{2 \times 2}} \mathcal{D}^{2-\alpha}\left\{\boldsymbol{e}(\boldsymbol{t})_{2 x 1}\right\}+ \\
& +\boldsymbol{k}_{\boldsymbol{d}_{2 \times 2}} \mathcal{D}^{\alpha+2}\left\{\boldsymbol{e}(\boldsymbol{t})_{2 x 1}\right\} .
\end{aligned}
$$

Taking twice derivative of the error function, (30) can be further written as follows

$$
\begin{aligned}
\boldsymbol{s}(\boldsymbol{t})_{2 \times 1}=\boldsymbol{k}_{p_{2 \times 2}} & \left\{\boldsymbol{x}_{\boldsymbol{d} 2 \times 1}-\boldsymbol{x}_{2 \times 1}\right\}+\boldsymbol{k}_{\boldsymbol{i}_{2 \times 2}} \mathcal{D}^{2-\alpha}\left\{\boldsymbol{e}(\boldsymbol{t})_{2 \times 1}\right\}+ \\
+ & \boldsymbol{k}_{\boldsymbol{d}_{2 \times 2}} \mathcal{D}^{\alpha+2}\left\{\boldsymbol{e}(\boldsymbol{t})_{2 \times 1}\right\} .
\end{aligned}
$$

Substituting $\boldsymbol{q}_{2 x 1}$ from (26) for (30), yields

$$
\begin{gathered}
\boldsymbol{s}(\boldsymbol{t})_{2 x 1}=\boldsymbol{k}_{p_{2 \times 2}}\left(\boldsymbol{x}_{d 2 \times 1}-\boldsymbol{f}(\boldsymbol{x})_{2 x 1}-\boldsymbol{g}(\boldsymbol{x})_{2 \times 2} \boldsymbol{u}(\boldsymbol{t})_{2 x 1}-\boldsymbol{\zeta}(\boldsymbol{t}, \boldsymbol{u}(\boldsymbol{t}))_{2 \times 1}\right)+ \\
+\boldsymbol{k}_{d_{2 \times 2}} \mathcal{D}^{\alpha+2}\left\{\boldsymbol{e}(\boldsymbol{t})_{2 x 1}\right\}+\boldsymbol{k}_{i_{2 \times 2}} \mathcal{D}^{2-\alpha}\left\{\boldsymbol{e}(\boldsymbol{t})_{2 \times 1}\right\}
\end{gathered}
$$

As it is clear that if $s(t)_{2 x 1}, \dot{s}(t)_{2 x 1}$, and $s(t)_{2 x 1}$ are convergence to zero in the sliding surface condition, then the tracking error $\left(\boldsymbol{e}(\boldsymbol{t})_{2 \times 1}\right)$ also goes to zero. By this convergence, considering the $s(t)_{2 x 1}=0$, the reaching phase control law $\left(\boldsymbol{u}_{\boldsymbol{r} p_{2 \times 1}}\right)$ is obtained as follows 


$$
\begin{gathered}
\boldsymbol{u}_{\boldsymbol{r} p_{2 \times 1}}=\boldsymbol{g}(\boldsymbol{x})_{2 \times 2}^{-1}\left(\boldsymbol{x}_{\boldsymbol{d} 2 \times 1}-\boldsymbol{f}(\boldsymbol{x})_{2 x 1}-\boldsymbol{\zeta}(\boldsymbol{t}, \boldsymbol{u}(\boldsymbol{t}))_{2 x 1}+\right. \\
\left.+\frac{\boldsymbol{k}_{\boldsymbol{d}_{2 \times 2}}}{\boldsymbol{k}_{p_{2 \times 2}}} \mathcal{D}^{\alpha+2}\left\{\boldsymbol{e}(\boldsymbol{t})_{2 x 1}\right\}+\frac{\boldsymbol{k}_{\boldsymbol{i}_{2 \times 2}}}{\boldsymbol{k}_{p_{2 \times 2}}} \mathcal{D}^{2-\alpha}\left\{\boldsymbol{e}(\boldsymbol{t})_{2 x 1}\right\}\right) .
\end{gathered}
$$

However, to control the system in terms of only considering the reaching phase control is not an effective way. So, as to guarantee that the proposed system is more effective against system disturbances, the switching control law, $\boldsymbol{u}_{s c_{2 \times 1}}$, should be added to the control signal

$$
u_{s c_{2 \times 1}}=g(x)_{2 x 2}^{-1}\left\{\lambda_{2 x 2} s(t)_{2 x 1}+\mu_{2 x 2} \operatorname{sign}\left(\dot{s}(t)_{2 x 1}\right)\right\}
$$

In (34), $\lambda_{2 x 2}$ and $\boldsymbol{\mu}_{2 x_{2}}$ show the switching gains, respectively. Thus, the total feedback control law, $\left(\boldsymbol{u}_{\text {total }_{2 \times 1}}\right)$, is written as follows

$$
\begin{gathered}
\boldsymbol{u}_{\text {total }_{2 \times 1}}=\boldsymbol{g}(\boldsymbol{x})_{2 \times 2}^{-1}\left(\boldsymbol{x}_{d 2 \times 1}-\boldsymbol{f}(\boldsymbol{x})_{2 x 1}-\right. \\
-\boldsymbol{\zeta}(\boldsymbol{t}, \boldsymbol{u}(\boldsymbol{t}))_{2 \times 1}+\frac{\boldsymbol{k}_{d_{2 \times 2}}}{\boldsymbol{k}_{p_{2 \times 2}}} \mathcal{D}^{\alpha+2}\left\{e(t)_{2 x 1}\right\}+ \\
+\frac{\boldsymbol{k}_{i_{2 \times 2}}}{\boldsymbol{k}_{p_{2 \times 2}}} \mathcal{D}^{2-\alpha}\left\{e(t)_{2 x 1}\right\}+\lambda_{2 x 2} s(t)_{2 x 1}+ \\
\left.+\mu_{2 \times 2} \operatorname{sign}\left(\dot{s}(t)_{2 x 1}\right)\right) .
\end{gathered}
$$

\section{EXPERIMENTAL RESULTS}

The FF-PIDSMC and the classical PI controller have been considered in terms of both tanks experimentally. The experimental setup is illustrated in Fig. 3 .

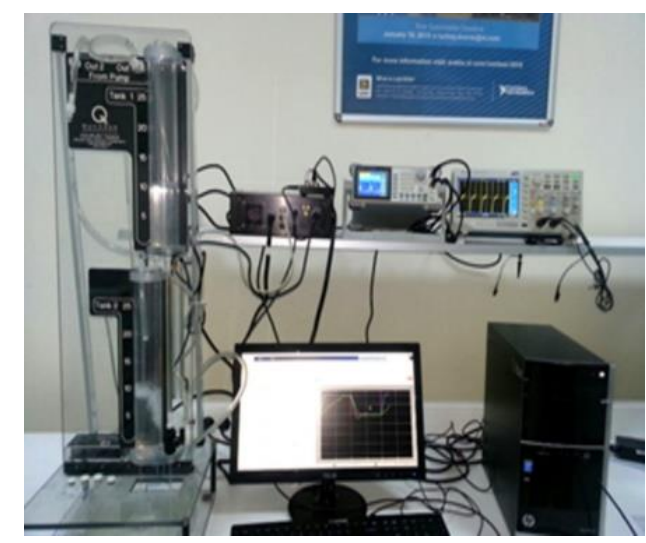

Fig. 3. The experimental setup system.

The controller has been created on SIMULINK. The Q8 DAQ device from Quanser Company has been run to perform real-time executions. The sampling time for the setup has been adjusted to $0.001 \mathrm{~s}$.

To test the controller's efficiency, a step and the sawtooth reference signal are combined and used together on both tank configurations. This kind of signal is very crucial for liquid level process control systems to obtain the system's performance. In this experiment, a step reference signal runs for $15 \mathrm{~s}$, and then a sawtooth reference signal runs for $45 \mathrm{~s}$ during the real-time execution. Figures from 4 to 6 show the liquid level trajectory tracking and controller signal of the proposed FO-PIDSMC controller, respectively. As seen from Fig. 4, the proposed FO-PIDSMC provides a pretty fast and effective output during the real-time execution, in addition to this, performing an acceptable small overshoot on the trajectory and precise tracking performance as compared with the classical FF-PI controller.

To verify the performance of the proposed FO-PIDSMC, the mean squared error (MSE) values are calculated and tabulated in Table II. The maximum peak overshoot value $\left(M_{p}\right)$ of the FF-PI controller is greater than that of the FOPIDSMC controller, and the mean squared error (MSE) value of the FF-PI controller is greater than that of the FOPIDSMC controller.

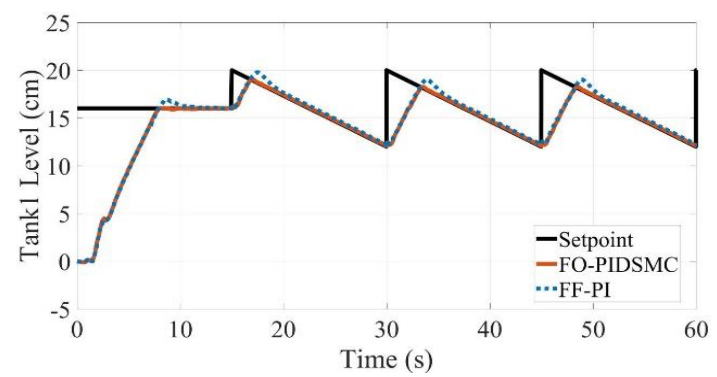

Fig. 4. Step + sawtooth responses for Tank 1 configuration.

TABLE II. SUMMARY OF EXPERIMENTAL RESULT FOR TANK 1.
\begin{tabular}{|c|c|c|}
\hline Controller & $\boldsymbol{M}_{\boldsymbol{p}}$ & MSE \\
\hline FF-PI & $\% 5,89$ & 17,0269 \\
\hline FO-PID-SMC & $\% 0,31$ & 12,0564 \\
\hline
\end{tabular}

The error of the Tank 1 level in the real-time process can be defined as the error between the given reference and the real-time measured trajectories. In Fig. 5, the maximum error percentage of the step and sawtooth is negligible for the proposed FF-PIDSMC and the maximum error percentage of the step and sawtooth reference is about $5.89 \%$ for the FF-PI.

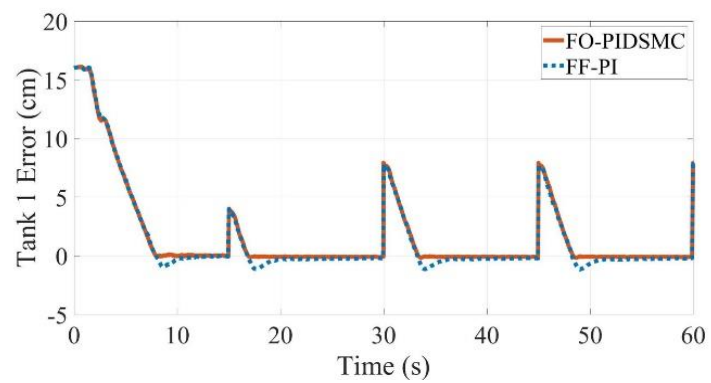

Fig. 5. Tracking error for Tank 1 configuration.

The reason for the outperformed results during the realtime process is that the FO-PIDSMC control signal is more robust and effective than that of the FO-PI (Fig. 6) thanks to combining with SMC control and FO calculus techniques.

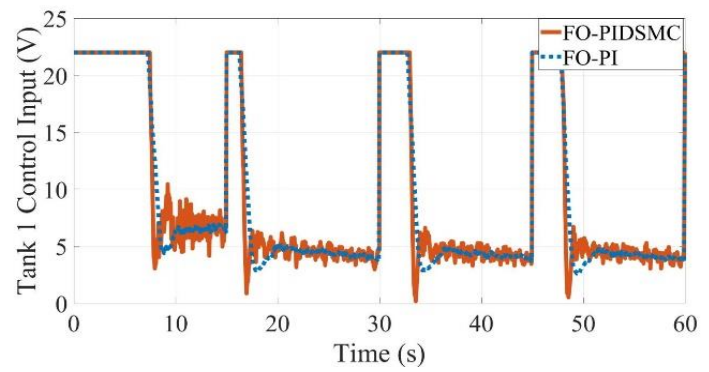

Fig. 6. Control inputs for Tank 1 configuration. 
Lastly, the same liquid level reference trajectory is also performed for coupled Tank 2 due to verify the FOPIDSMC's efficiency in coupled Tank 2. As shown in Fig. 7 , the proposed FO-PIDSMC gives a quite fast response with a small overshoot in trajectory. In this experimental setup, the proposed controller of Tank 2 computes the reference liquid in Tank 1, while the other controller computes the real-time system's pump voltage command to guarantee the real-time liquid level in Tank 2.

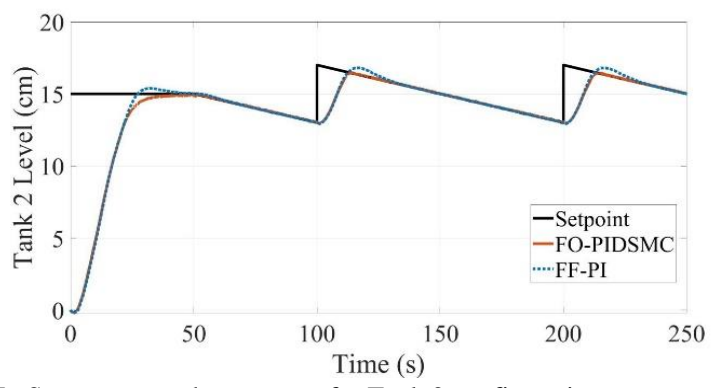

Fig. 7. Step + sawtooth responses for Tank 2 configuration.

In Fig. 8, the maximum error percentage of the step and sawtooth is negligible for the proposed FF-PIDSMC and the maximum error percentage of the step and sawtooth reference is about $2,54 \%$ for the FF-PI.

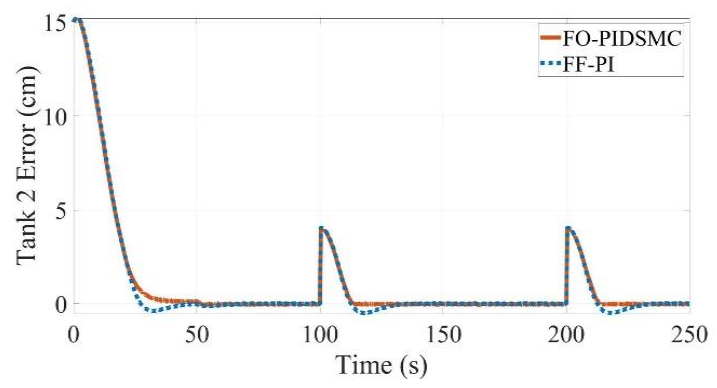

Fig. 8. Tracking error for Tank 2 configuration.

As also seen in Fig. 9, the outperformed results during the real-time process is that the FO-PIDSMC control signal is more robust and effective than that of the FO-PI. Similarly, to show the efficiency of the proposed controller, the MSE values in terms of the level values of Tank 2 are given in Table III. The real-time results verify that FO-PIDSMC works well for Tank 2. As shown in Table III, the $M_{p}$ of the proposed controller is less when compared with the FF-PI controller. In conclusion, the findings of this real-time study demonstrate that the proposed controller gives an efficient real-time trajectory tracking response compared to the FF-PI controller and the precision of the coupled tank system is quite effective.

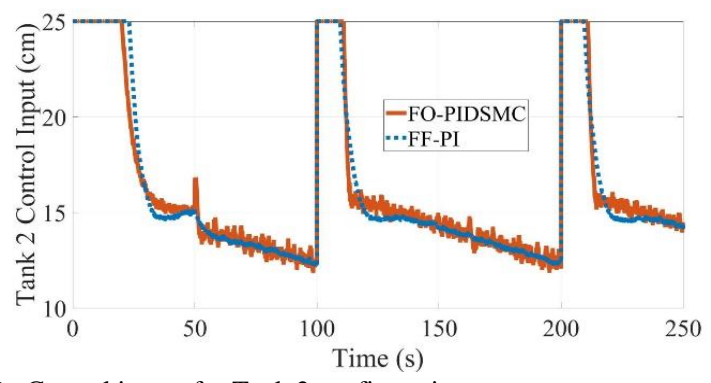

Fig. 9. Control inputs for Tank 2 configuration.
TABLE III. SUMMARY OF EXPERIMENTAL RESULT FOR TANK 2.

\begin{tabular}{|c|c|c|}
\hline Controller & $\boldsymbol{M}_{\boldsymbol{p}}$ & MSE \\
\hline FF-PI & $\% 2,54$ & 10,1834 \\
\hline FO-PIDSMC & $\% 0,13$ & 7,2543 \\
\hline
\end{tabular}

\section{CONCLUSIONS}

In this experimental application, the proposed FOPIDSMC technique has been given to control effectively the uncertain non-linear liquid level coupled tank system in undesired circumstances. In terms of performing accurate trajectory tracking, finite-time convergence, and chatteringfree control inputs, the FO-PIDSMC control technique is presented. By combining FO calculus with the SMC control technique, the effect of the measures' disturbance is compensated. The experimental results proved that the FOPIDSMC gives a remarkable real-time liquid level trajectory tracking performance during the experimental process. Based on the experimental results (Tank 1), MSE error is decreased about $40 \%$, and MSE error is also decreased about $50 \%$ for Tank 2. Owing to the FO calculus action of the proposed control technique, the FO-PIDSMC controller follows the given liquid level reference trajectory with a very small error when compared with the responses of the FF-PI controller.

\section{CONFLICTS OF INTEREST}

The authors declare that they have no conflicts of interest.

\section{REFERENCES}

[1] G. S. Sankar, S. M. Kumar, S. Narasimhan, S. Narasimhan, and S. M Bhallamudi, "Optimal control of water distribution networks with storage facilities", Journal of Process Control, vol. 32, pp. 127-137, 2015. DOI: 10.1016/j.jprocont.2015.04.007.

[2] R. Boiocchi, M. Mauricio-Iglesias, A. K. Vangsgaard, K. Gernaey, and G. Sin, "Aeration control by monitoring the microbiological activity using fuzzy logic diagnosis and control. Application to a complete autotrophic nitrogen removal reactor", Journal of Process Control, vol. 30, pp. 22-33, 2015. DOI 10.1016/J.JPROCONT.2014.10.011.

[3] W. Qi, J. Liu, and P. D. Christofides, "A distributed control framework for smart grid development: Energy/water system optimal operation and electric grid integration", Journal of Process Control, vol. 21, no. 10, pp. 1504-1516, 2011. DOI 10.1016/j.jprocont.2011.05.010.

[4] T. Xu, H. Yu, J. Yu, and X. Meng, “Adaptive disturbance attenuation control of two tank liquid level system with uncertain parameters based on Port-Controlled Hamiltonian", IEEE Access, vol. 8, pp. 47384-47392, 2020. DOI: 10.1109/ACCESS.2020.2979352.

[5] R. Sotner, O. Domansky, J. Jerabek, N. Herencsar, J. Petrzela, and D. Andriukaitis, "Integer-and Fractional-Order Integral and Derivative Two-Port Summations: Practical Design Considerations," Applied Sciences, vol. 10, no. 1, p. 54, Dec. 2019. DOI: 10.3390/app10010054

[6] K. Sundaravadivu, V. Jeyakumar, and K. Saravanan, "Design of Fractional Order PI controller for liquid level control of spherical tank modeled as Fractional Order System", in Proc. of 2011 IEEE International Conference on Control System, Computing and Engineering, 2011, pp. 522-525. DOI: 10.1109/ICCSCE.2011.6190581.

[7] L. Langhammer, R. Sotner, J. Dvorak, J. Jerabek and D. Andriukaitis, "Reconnection-less Reconfigurable Fractional-Order Current-Mode Integrator Design With Simple Control" IEEE Access, vol. 9, pp. 136395-136405, 2021. Doi: 10.1109/ACCESS.2021.3117016.

[8] W. Zhang, S. Li, and Y. Liu, "Adaptive sliding mode back-stepping speed control of hydraulic motor for wave energy conversion device", IEEE Access, vol. 8, pp. 89757-89767, 2020. DOI: 10.1109/ACCESS.2020.2992708.

[9] H. Gouta, S. H. Said, N. Barhoumi, and F. M'Sahli, "Observer-based back-stepping controller for a state-coupled two-tank system", IETE Journal of Research, vol. 61, no. 3, pp. 259-268, 2015. DOI: 
10.1080/03772063.2015.1018846.

[10] L. Liang, "The application of fuzzy PID controller in coupled-tank liquid-level control system", in Proc. of 2011 International Conference on Electronics, Communications and Control (ICECC), 2011, pp. 2894-2897. DOI: 10.1109/ICECC.2011.6067785.

[11] R. Jain, N. Sivakumaran, and T. K. Radhakrishnan, "Design of self tuning fuzzy controllers for nonlinear systems", Expert Systems with Applications, vol. 38, no. 4, pp. 4466-4476, 2011. DOI: 10.1016/j.eswa.2010.09.118.

[12] S. T. Lian, K. Marzuki, and Y. Rubiyah, "Tuning of a neuro-fuzzy controller by genetic algorithms with an application to a coupled-tank liquid-level control system", Engineering Applications of Artificial Intelligence, vol. 11, no. 4, pp. 517-529, 1998. DOI: 10.1016/S09521976(98)00012-8.

[13] F. Behrooz, N. Mariun, M. H. Marhaban, M. A. M. Radzi, and A. R. Ramli, "Review of control techniques for HVAC systemsNonlinearity approaches based on fuzzy cognitive maps", Energies, vol. 11, no. 3, p. 495, 2018. DOI: 10.3390/en11030495.

[14] S. Mobayen, "A novel global sliding mode control based on exponential reaching law for a class of under-actuated systems with external disturbances", Journal of Computational and Nonlinear Dynamics, vol. 11, no. 2, pp. 11-21, 2016. DOI: 10.1115/1.4031087.

[15] S. Mobayen, "An adaptive fast terminal sliding mode control combined with global sliding mode scheme for tracking control of uncertain nonlinear third-order systems", Nonlinear Dynamics, vol. 82, no. 1, pp. 599-610, 2015. DOI: 10.1007/s11071-015-2180-4.

[16] S. H. Zak and S. Hui, "On variable structure output feedback controllers for uncertain dynamic systems", IEEE Trans. on Automatic Control, vol. 38, no. 10, pp. 1509-1512, 1993. DOI: 10.1109/9.241564.

[17] C. Edwards and S. K. Spurgeon, "Sliding mode stabilization of uncertain systems using only output information", International Journal of Control, vol. 62, no. 5, pp. 1129-1144, 1995. DOI: $10.1080 / 00207179508921587$.

[18] C. Mu and H. He, "Dynamic behavior of terminal sliding mode control", IEEE Transactions on Industrial Electronics, vol. 65, no. 4 pp. 3480-3490, Apr. 2018. DOI: 10.1109/TIE.2017.2764842.

[19] G. Bartolini, E. Punta, and T. Zolezzi, "Approximability properties for second-order sliding mode control systems", IEEE Transactions on Automatic Control, vol. 52, no. 10, pp. 1813-1825, Oct. 2007. DOI: 10.1109/TAC.2007.906179.

[20] K. Ma, "Comments on "Quasi-continuous higher order sliding-mode controllers for spacecraft-attitude-tracking maneuvers"”, IEEE Transactions on Industrial Electronics, vol. 60, no. 7, pp. 2771-2773, Jul. 2013. DOI: 10.1109/TIE.2012.2196012.

[21] Q.-Y. Fan and G.-H. Yang, "Adaptive actor-critic design-based integral sliding-mode control for partially unknown nonlinear systems with input disturbances", IEEE Transactions on Neural Networks and Learning Systems, vol. 27, no. 1, pp. 165-177, Jan. 2016. DOI:
10.1109/TNNLS.2015.2472974

[22] D. Liu and G. Yang, "Prescribed performance model-free adaptive integral sliding mode control for discrete-time nonlinear systems", IEEE Transactions on Neural Networks and Learning Systems, vol. 30, no. 7, pp. 2222-2230, Jul. 2019. DOI: 10.1109/TNNLS.2018.2881205.

[23] Q.-D. Hoang, V. A. Rosas-Cervantes, S.-G. Lee, I.-S. Weon, J.-H Choi, and Y.-H. Kwon, "Robust finite-time convergence control mechanism for high-precision tracking in a hybrid fluid power actuator", IEEE Access, vol. 8, pp. 196775-196789, 2020. DOI: 10.1109/ACCESS.2020.3033799.

[24] M. Ö. Efe, "Fractional fuzzy adaptive sliding-mode control of a 2DOF direct-drive robot arm", IEEE Transactions on Systems, Man, and Cybernetics, Part B (Cybernetics), vol. 38, no. 6, pp. 1561-1570, 2008. DOI: 10.1109/TSMCB.2008.928227.

[25] Y. Wang, L. Gu, Y. Xu, and X. Cao, "Practical tracking control of robot manipulators with continuous fractional-order nonsingular terminal sliding mode", IEEE Transactions on Industrial Electronics, vol. 63, no. 10, pp. 6194-6204, 2016. DOI 10.1109/TIE.2016.2569454.

[26] M. N. Soorki and M. S. Tavazoei, "Adaptive robust control of fractional-order swarm systems in the presence of model uncertainties and external disturbances", IET Control Theory \& Applications, vol 12, no. 7, pp. 961-969, 2018. DOI: 10.1049/iet-cta.2017.0035.

[27] M. Bataghva and M. Hashemi, "Adaptive sliding mode synchronisation for fractional-order non-linear systems in the presence of time-varying actuator faults", IET Control Theory \& Applications, vol. 12 , no. 3, pp. 377-383, 2018. DOI: 10.1049/IETCTA.2017.0458

[28] R. Li, F. Wu, P. Hou, and H. Zou, "Performance assessment of FOPID temperature control system using a fractional order LQG benchmark", IEEE Access, vol. 8, pp. 116653-116662, 2020. DOI 10.1109/ACCESS.2020.3004701.

[29] P. P. Arya and S. Chakrabarty, "A robust internal model-based fractional order controller for fractional order plus time delay processes", IEEE Control Systems Letters, vol. 4, no. 4, pp. 862-867, Oct. 2020. DOI: 10.1109/LCSYS.2020.2994606

[30] I. Birs, C. Muresan, I. Nascu, and C. Ionescu, "A survey of recent advances in fractional order control for time delay systems", IEEE Access, vol. 7, pp. 30951-30965, 2019. DOI: 10.1109/ACCESS.2019.2902567.

[31] Y. Teng, H. Li, and F. Wu, "Design of distributed fractional order PID type dynamic matrix controller for large-scale process systems", IEEE Access, vol. 8, pp. 179754-179771, 2020. DOI 10.1109/ACCESS.2020.3027597.

[32] M. A. Louis, M. R. Roman, O. E. Mahmoud, and M. F. Sedrak, "Performance investigation and control parameters choice for sliding mode control of coupled tanks system", in IOP Conf. Ser.: Mater. Sci. Eng., 2020, vol. 973. DOI: 10.1088/1757-899X/973/1/012044.

This article is an open access article distributed under the terms and conditions of the Creative Commons Attribution 4.0 (CC BY 4.0) license (http://creativecommons.org/licenses/by/4.0/). 